\title{
Special issue on microscopy image analysis for biomedical applications
}

\author{
Stephen J. McKenna - Derek Magee - Nasir M. Rajpoot
}

Published online: 8 June 2012

(C) Springer-Verlag 2012

Demand for tools that extract quantitative information from microscopy images of biological samples continues to grow. This is providing interesting challenges for computer vision researchers. Typical tasks are detection, segmentation, classification, motion analysis, and tracking of cells and subcellular compartments. Image data are acquired using various imaging technologies, each with its own characteristics. In the six papers presented in this special issue these include brightfield [1], phase contrast [7], electron [5], and fluorescence $[2,3,6]$ microscopy. There is also considerable variation in the biological samples to be analysed. Here these include plant roots, cervical cancer cells and neuronal dendrites, for example.

These papers were selected and revised from submissions received in response to an open call for papers. Three of them $[1,5,6]$ were developed from presentations at the symposium on microscopy image analysis for biomedical applications that we chaired in April 2010. The meeting was organised by the British Machine Vision Association (BMVA) and held at the British Computer Society in London. A further paper based on a talk given at that meeting, on nuclei detection using support vector machines, has also been published recently in this journal [4].

S. J. McKenna ( $\varangle)$

School of Computing, University of Dundee,

Dundee DD1 4HN, UK

e-mail: stephen@computing.dundee.ac.uk

D. Magee

School of Computing, University of Leeds, Leeds LS2 9JT, UK

e-mail: d.r.magee@leeds.ac.uk

N. M. Rajpoot

Department of Computer Science, University of Warwick, Coventry CV4 7AL, UK

e-mail: n.m.rajpoot@warwick.ac.uk
Automatic analysis of adherent mammalian cells in brightfield images is a challenging task, not least because of their low contrast. It is a problem that has arguably received relatively little attention in the literature. Ali et al. [1] present methods for detection and segmentation of adherent cells in brightfield images using phase and texture features. As a side benefit, their framework allows registration of cells in brightfield and fluorescence images. They evaluate their methods by comparison to manual and fluorescence-based segmentation. Their software sephaCe is open-source and has been made freely available.

Esteves et al. [3] also address detection and segmentation but in a rather different setting: analysis of cell nuclei in confocal fluorescence images of Arabidopsis thaliana root tips. They demonstrate that an improved gradient convergence filter method allows shape priors to be imposed on this largely bottom-up process. This improves nucleus detection in scenarios where cells appear overlapping or in close proximity.

Sethuraman et al. [6] also analyse confocal fluorescence images of A. thaliana but are concerned with tracking cell membranes rather than segmenting nuclei. These membranes appear in a given two-dimensional slice as a network structure. Their contribution is to propose a Markov chain Monte Carlo sampling scheme for tracking this structure using network snakes.

Theriault et al. [7] segment, classify and track mouse fibroblast cells imaged using phase-contrast microscopy. They employ a battery of standard shape and appearance-based features to characterise their morphological state. They use AdaBoost to classify each cell instance into one of eight classes based on spread, polarization and orientedness attributes. They also show that the performance of their simple tracking algorithm increases as they use the classification algorithm to filter out substrate clutter. Expert-labeled datasets for timelapse phase-contrast microscopy images of cells are scarce, 
and so it is a welcome development that the authors have promised to make their data available to the wider image analysis research community interested in developing and validating algorithms for detection, classification and tracking of live cells. Such algorithms will be important for studying the biological properties of cells in migration under different conditions.

Delpiano et al. [2] adopt a different approach to motion analysis. Rather than tracking cells, they employ optical flow techniques to analyse the movement of fluorescent point sources. They perform evaluation using both synthetic data and images of hippocampal neuronal dendrites.

Finally, Hermann et al. [5] describe a fully automated system for high-throughput screening of two-dimensional crystallization experiments using transmission electron microscopy. Their software automatically explores and analyses samples deposited on carbon-coated grids at several magnifications. Image analysis is used to identify interesting regions that are then acquired and analysed at higher magnifications.

We would like to thank the reviewers for their evaluations, the authors for carefully revising their papers, as well as all those who helped to make the original BMVA meeting a success.

\section{References}

1. Ali, R., Gooding, M., Szilágyi, T., Vojnovic, B., Christlieb, M., Brady, M.: Automatic segmentation of adherent biological cell boundaries and nuclei from brightfield microscopy images. Mach. Vis. Appl. (2012). doi:10.1007/s00138-011-0337-9

2. Delpiano, J., Jara, J., Scheer, J., Ramírez, O.A., Ruiz-del Solar, J., Härtel, S.: Performance of optical flow techniques for motion analysis of fluorescent point signals in confocal microscopy. Mach. Vis. Appl. (2012). doi:10.1007/s00138-011-0362-8

3. Esteves, T., Quelhas, P., Mendonça, A.M., Campilho, A.: Gradient convergence filters and a phase congruency approach for in vivo cell nuclei detection. Mach. Vis. Appl. (2012). doi:10.1007/s00138012-0407-7

4. Han, J.W., Breckon, T.P., Randell, D.A., Landini, G.: The application of support vector machine classification to detect cell nuclei for automated microscopy. Mach. Vis. Appl. (2011). doi:10.1007/ s00138-010-0275-y

5. Hermann, G., Coudray, N., Buessler, J.L., Caujolle-Bert, D., Rémigy, H.W., Urban, J.P.: ANIMATED-TEM: a toolbox for electron microscope automation based on image analysis. Mach. Vis. Appl. (2012). doi:10.1007/s00138-011-0357-5
6. Sethuraman, V.S., French, A.P., Wells, D.M., Kenobi, K., Pridmore, T.P.: Tissue-level segmentation and tracking of cells in growing plant roots. Mach. Vis. Appl. (2012). doi:10.1007/s00138-011-0329-9

7. Theriault, D.H., Walker, M., Wong, J.Y., Betke, M.: Cell morphology classification and clutter mitigation in phase-contrast microscopy images using machine learning. Mach. Vis. Appl. (2012). doi:10. 1007/s00138-011-0345-9

\section{Author Biographies}

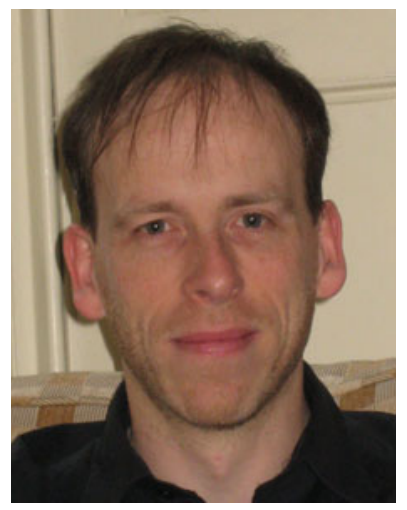

Stephen J. McKenna is Chair of Computer Vision in the School of Computing, University of Dundee. After graduating in computer science from the University of Edinburgh (1990) and obtaining Ph.D from the University of Dundee (1994), he held postdoctoral research positions at Queen Mary, University of London and Tecnopolis Csata, Italy. He has also been a visiting researcher at BT Labs and George Mason University. His research interests include the development and application of computer vision, pattern recognition and machine learning methods in domains such as intelligent humancomputer interaction, content-based image browsing, medicine and biology. Funders of his research have included EPSRC, BBSRC, MRC, TSB and CSO. He co-authored the book Dynamic Vision and has published more than 100 articles. He is an associate editor for the journals Machine Vision and Applications and Pattern Analysis and Applications.

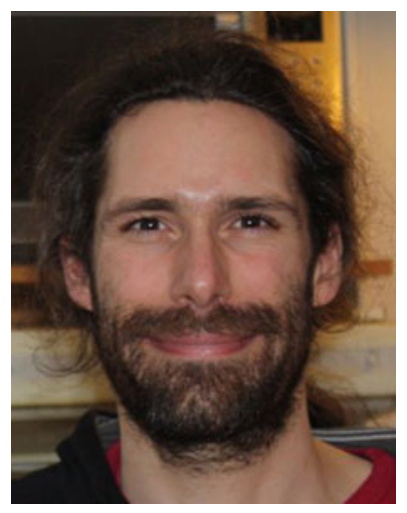

Dr Derek Magee is a lecturer in the School of Computing at the University of Leeds, UK, where previously he held a 5-year University Research Fellowship. He completed his $\mathrm{Ph} . \mathrm{D}$ on visual livestock gait analysis at Leeds under Roger Boyle in 2000, winning the Industry prize at the British Machine Vision Conference for this work that year. His subsequent research has mainly been in the field of medical image analysis and medical simulation, although he maintains interests in machine learning (winning the 2004 BCS Machine Intelligence prize) and sports analysis (developing commercial software that has been used for the analysis of English Premiership football). 


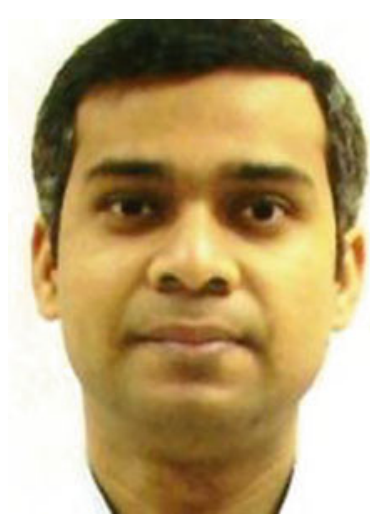

Nasir M. Rajpoot graduated with a Ph.D in digital image coding from the University of Warwick in 2001. During 19982000, he was a visiting postgraduate research fellow at the Applied Mathematics programme of Yale University. Prior to that, he completed his M.Sc in systems engineering and M.Sc in computer science from Quaid-eAzam University and Bahauddin Zakariya University in Pakistan in 1996 and 1994, respectively. Dr Rajpoot currently holds the position of Associate Professor in Computer Science at the University of Warwick, where he heads his laboratory in the Computational Biology and Bioimaging (COMBI) Research Group. The group is internationally renowned for its groundbreaking translational research in digital pathology and computational biology. A main focus of his research in recent years has been development of algorithms for computerized histopathology image analysis. His current research interests lie in the areas of bioimage computing, histopathology image analysis, image processing and pattern recognition. Dr Rajpoot has published 3 edited books, over 60 refereed articles in the areas of image coding and denoising, texture analysis and shape analysis. He is on the review panels of more than a dozen international journals and on the programme committee of a number of international conferences. He was the General Chair of Medical Image Understanding and Analysis (MIUA) and the Technical Chair of British Machine Vision Conference (BMVC), both held at Warwick in July 2010 and September 2007, respectively. 